\title{
Viscous Normal Shock Solutions Including Chemical, Thermal, and Radiative Nonequilibrium
}

\author{
David R. Mott* \\ University of Michigan, Ann Arbor, Michigan 48109-2118 \\ Thomas A. Gally \\ American University in Cairo, 11511 Cairo, Egypt \\ and \\ Leland A. Carlson $\$$ \\ Texas A\&M University, College Station, Texas 77843-3141
}

\begin{abstract}
An existing axisymmetric body viscous shock layer code including thermochemical and thermodynamic nonequilibrium and nonequilibrium radiative gasdynamic coupling was adapted to simulate the one-dimensional flow within a shock tube. A suitable solution scheme for this case and additional radiation modeling were developed in order to compare the current computational results with experimental radiation measurements. Spectrally integrated intensity traces, time to peak radiation, and ionization distance data were generated for shocks in air with speeds between $9.5-12.6 \mathrm{~km} / \mathrm{s}$. Using the current model, the dual peak characteristics of Wilson's experimental results are reproduced without the introduction of contaminant radiation. Overall, good agreement is seen between the current calculations and the available experimental data, justifying the use of the current nonequilibrium models for engineering applications.
\end{abstract}

\section{Nomenclature}

$A=$ vibrational model parameter in Eq. (7)

$C=$ mass fraction

$c_{1}=$ constant defined by Eq. (1)

$c_{p}=$ constant pressure specific heat

$d_{i}=$ ionization distance

$E=$ vibrational energy lost in dissociation

$=$ internal energy

$G=$ vibrational energy gained in recombination

$h=$ specific enthalpy

$I=$ radiation intensity

$J=$ diffusive mass flux

$k=$ thermal conductivity

$M_{i}=$ molecular weight of species $i$

$p=$ pressure

$\mathscr{R}=$ universal gas constant

$r=$ coordinate perpendicular to the tube wall

$\dot{q}_{r}=$ streamwise radiative heat flux

$S=$ radiant source function

$T_{e}=$ electron-electronic temperature

$T_{i}=$ translational-rotational temperature of species $i$

$T_{1}=$ vibrational temperature

$t=$ time coordinate

$U=$ diffusion velocity

$\gamma=$ velocity

$\dot{w}=$ chemical production term

$y=$ coordinate parallel to the shock tube

$\Delta=$ downstream boundary distance

$\delta \Phi=$ calculated change in $\Phi$ for one iteration

Presented as Paper 94-2415 at the AIAA 25th Plasmadynamics and Laser Conference, Colorado Springs, CO, June 20-23, 1994; received July 25, 1994; revision received Jan. 23, 1995; accepted for publication Jan. 24, 1995. Copyright (c) 1994 by the American Institute of Aeronautics and Astronautics, Inc. All rights reserved.

${ }^{*}$ Graduate Student, Department of Aerospace Engineering. Member AIAA

$\dagger$ Assistant Professor, Department of Mechanical Engineering, 113 Sharia Kasr el Aini, P.O. Box 2511. Member AIAA.

末Professor, Department of Aerospace Engineering. Associate Fellow AIAA.

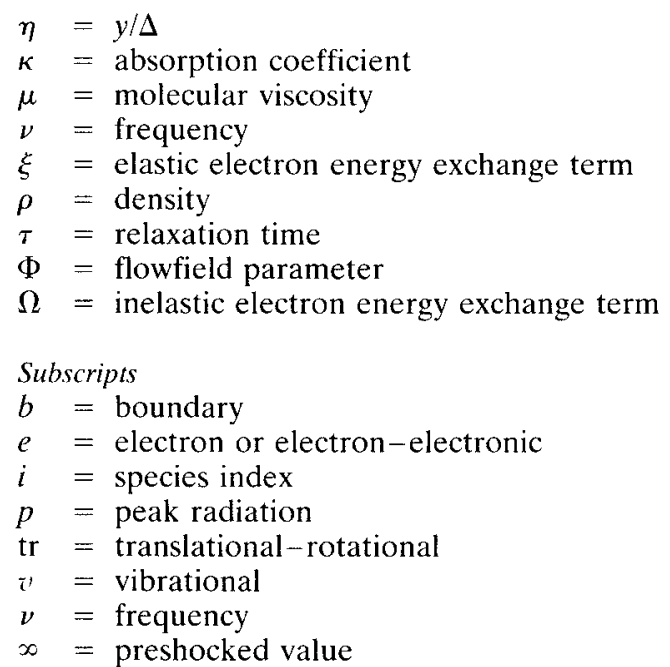

\section{Introduction}

A EROBRAKING vehicles have received much attention recently in terms of interorbital or interplanetary transfer missions. The aerobraking maneuver is characterized by a prolonged flight at very low densities and very high velocities; previously designed vehicles spent only short durations at these low-density altitudes. Accurate prediction of the environment in which aerobraking vehicles operate requires the development of engineering tools for modeling the chemical, thermal, and radiative nonequilibrium present in such lowdensity flows.

In the past, research efforts have produced engineering models for predicting the shock layer flowfield properties including nonequilibrium about an axisymmetric body. ${ }^{1} \mathrm{Re}-$ cently, building upon a version of the viscous shock layer (VSL) solver SHTNEQ code developed at NASA Langley, ${ }^{2}$ these solvers have been extended to include ronequilibrium radiation modeling and a three-temperature (vibrational $T_{i,}$, translational-rotational $T$, and electron-electronic $T_{e}$ ) gas. ${ }^{3-6}$ The viscous shock layer equations are an approximation to the Navier-Stokes equations, and the solution 
methodology for solving these equations for axisymmetric and nonaxisymmetric flow is detailed in Refs. 7 and 8 . Calculations performed by this improved model have been compared to stagnation point experimental data from the Fire II flight test ${ }^{9}$ with good results. ${ }^{3,10,11}$

Since experimental data in which nonequilibrium and radiative phenomena occur simultaneously is limited, all possible avenues for model validation must be pursued. While the Fire II flight experiment provided a limited amount of radiative heat transfer and surface information for the stagnation point of an axisymmetric body, most information for nonequilibrium flow is available in the form of shocktube data. Shock-tube experiments have produced shocks in which nonequilibrium processes and radiation are important, making such experiments vital in the verification of nonequilibrium chemical and radiation models. ${ }^{12-19}$ Several investigators have attempted to recreate these experiments computationally with models of varying complexity, ${ }^{19-22}$ with varying degrees of success.

The current work presents the conversion of the axisymmetric nonequilibrium flow code previously described to a form suitable for simulating shock-tube experiments. Calculated results for the nonequilibrium flowfield are then compared with experimental data in order to validate the current nonequilibrium chemical and radiative models.

\section{Flowfield Theory}

The basic models employed in the current work are outlined in detail in the literature ${ }^{3.23-28}$; the nonequilibrium models have not been changed. The program has been converted to simulate the one-dimensional shock-tube problem. A detailed description of the entire one-dimensional solution procedure is given in Ref. 29. The following discussion outlines the primary concerns addressed in the conversion of the code and includes additional modeling required for calculating radiation data compatible with data measured in shock-tube experiments

The coordinate system utilized by the normal shock equations is shown in Fig. 1. This coordinate system may appear awkward at first, but recall that the normal shock code was developed from an existing axisymmetric body code. In the original code, the $y$ coordinate was normal to the body and went from $\eta=y / \Delta=0$ at the body surface to $\eta=1$ at the shock ( $\Delta$ being the shock standoff distance). The normal shock code maintains the shock at $\eta=1$, and $\eta=0$ corresponds to a point at a specified distance downstream of the shock.

\section{Conservation Equations}

Adding nonequilibrium effects to the flowfield model does not affect the mass and momentum conservation equations directly. One-dimensional mass conservation is given by Eq. (1):

$$
\rho v^{\prime}=\text { const }=\rho_{x_{x}} v_{x}=c_{1}
$$

The subscript $\propto$ denotes conditions prior to the shock. Under viscous shock layer assumptions, normal viscous stresses are neglected, reducing the one-dimensional momentum equation to the form in Eq. (2):

$$
p+\rho v^{2}=\text { const }=p_{\infty}+\rho_{\infty} v_{\infty}^{2}
$$

Using the mass conservation equation, Eq. (2) can be solved for the local pressure:

$$
p=p_{x}+c_{1}\left(v_{x}-v\right)
$$

The equation of state used in the current model follows a partial pressure formulation to account for the multicompo-

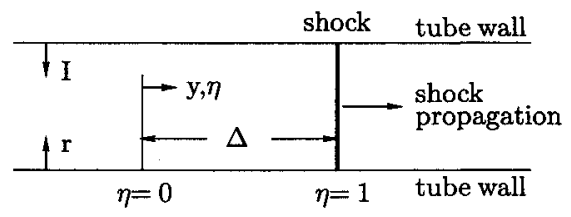

Fig. 1 Normal shock coordinate system.

nent nature of the gas. The form of the equation of state is given in Eq. (4):

$$
\rho=p / \mathscr{R} \sum_{i} \frac{C_{i}}{M_{i}} T_{i}
$$

In Eq. (4), $T_{i}$ is the translational temperature for species $i$. $T_{i}$ is $T$ unless the index $i$ denotes the free electrons in the flow; in that case, $T_{i}=T_{e}$.

The energy conservation equations and species continuity equations do not lead to simple algebraic formulas for the translational, vibrational, and electron-electronic temperatures and the species mass fractions. The one-dimensional forms of these equations are presented in Ref. 29 , and the details for modeling the various terms in each are given in Ref. 3. (5):

The species continuity equation for species $i$ is given in $\mathrm{Eq}$.

$$
\rho v \frac{\partial C_{i}}{\partial y}=\dot{w}_{i}-\frac{\partial J_{i}}{\partial y}
$$

The details for modeling the terms in Eq. (5) are given in Ref. 3. The chemistry model primarily uses the verified and accepted reaction rates of Park, with the exception of the electron-impact rates. These latter rates were derived from the detailed theory of Kunc and tested by comparison with Fire II results. ${ }^{4}$ The reaction set and the origin of the individual reaction rates are given in Refs. 3 and 4.

Global energy conservation is represented by Eq. (6):

$$
\begin{aligned}
& \rho \tau^{\prime}\left(c_{p, \mathrm{tr}} \frac{\partial T}{\partial y}+c_{p, \mathrm{u}} \frac{\partial T_{v}}{\partial y}+c_{p, e} \frac{\partial T_{c}}{\partial y}\right)-\frac{\partial}{\partial y}\left(k_{\mathrm{tr}} \frac{\partial T}{\partial y}+k_{i} \frac{\partial T_{v}}{\partial y}\right. \\
& \left.+k_{e} \frac{\partial T_{c}}{\partial y}\right)-\sum_{i} \rho_{i} U_{i}\left(c_{p \mathrm{tr}, i} \frac{\partial T}{\partial y}+c_{p r, i} \frac{\partial T_{i,}}{\partial y}+c_{p e, i} \frac{\partial T_{e}}{\partial y}\right) \\
& -\tau \frac{\partial p}{\partial y}+\frac{\partial}{\partial y}\left[-\frac{2}{3} v \mu \frac{\partial v}{\partial y}\right]+\sum_{i} \dot{w}_{i} h_{i}=-\frac{\partial \dot{q}_{r}}{\partial y}
\end{aligned}
$$

The first three terms account for energy convection, conduction, and diffusion, respectively, with each energy mode possessing an associated specific heat $c_{p}$ and thermal conductivity $k$. Pressure and viscous work terms are then included, followed by the chemical reaction term to account for the change in the zero-point energies of the species present. The $\dot{q}_{r}$ term is the streamwise radiant energy flux calculated by the nonequilibrium radiation model.

The vibrational energy model is that of Green. ${ }^{5.25}$ The vibrational energy equation is given in one-dimensional form as follows:

$$
\begin{aligned}
& \rho \tau c_{p, i} \frac{\partial T_{y,}}{\partial y}=\frac{\partial}{\partial y}\left(k_{z} \frac{\partial T_{z}}{\partial y}\right)+\sum_{i} \rho_{i} U_{i} c_{p v, i} \frac{\partial T_{i,}}{\partial y} \\
& +\sum_{i} \rho_{i} \frac{e_{i, i}\left(T_{c}\right)-e_{i i^{\prime} i}}{\tau_{c, i}}+\sum_{i} \rho_{i} A \frac{e_{i, i}(T)-e_{i^{\prime} i}}{\tau_{i}} \\
& +\sum_{i}\left(e_{x^{\prime}, i}-E_{i}\right)\left(\frac{\partial \rho_{i}}{\partial t}\right)_{f}-\sum_{i}\left(e_{i / i}-G_{i}\right)\left(\frac{\partial \rho_{i}}{\partial t}\right)_{b}
\end{aligned}
$$


The vibrational temperature $T_{i}$, represents the average vibrational energy per diatomic particle in the flow assuming a Boltzmanm distribution among vibrational energy levels. The last four terms on the RHS of Eq. (7) are terms that account for gains or losses in the vibrational energy due to transfer between the various energy modes. The third term accounts for the electron-vibrational energy exchange, and the last three terms account for translational-vibrational coupling through thermal and chemical exchange. In Eq. (7), $e_{v i}\left(T_{\mathrm{e}}\right)$ gives the vibrational energy per unit mass of species $i$ if the vibrational temperature were the electron-electronic temperature; energy transfer between the vibrational and electronic modes occurs when $e_{i}\left(T_{c}\right)$ differs from the actual vibrational energy $e_{r^{\prime},}$. An analogous energy exchange between the vibrational and translational modes is calculated using the fourth term on the RHS of Eq. (7). The final two terms in Eq. (7) give the vibrational energy lost due to dissociation and gained through recombinations.

Finally, the electron-electronic energy equation is given by Eq. (8):

$$
\begin{aligned}
& \rho^{\sigma} c_{p, e} \frac{\partial T_{e}}{\partial y}-\frac{\partial}{\partial y}\left(k_{e} \frac{\partial T_{e}}{\partial y}\right)-\sum_{i} \rho_{i} U_{i} c_{p e, i} \frac{\partial T_{e}}{\partial y} \\
& -v \frac{\partial p_{e}}{\partial y}+\dot{w}_{c} h_{\mathrm{tr}_{c}}+\sum_{i} \dot{w}_{i} h_{e_{i}} \\
& =-\sum_{i} \rho_{i} \frac{e_{v^{\prime} i}\left(T_{e}\right)-e_{i, j}}{\tau_{e, i}}+\sum_{i} \xi_{c i}+\Omega_{c}
\end{aligned}
$$

The free electron translational temperature and the electronic temperature are assumed to be in equilibrium and represented by the temperature $T_{1}$. As with the last four terms in the vibrational energy equation, the terms on the RHS of Eq. (8) are source terms used to account for energy transfer between the various energy modes. The first term calculates the exchange between vibrational and free electron energy, the $\xi_{c i}$ summation accounts for energy exchange due to elastic collisions between free electrons and the other particles in the field, and $\Omega_{c}$ represents energy transfer to and from free electrons due to inelastic, chemical processes.

\section{Solution Procedure}

As seen in the previous section, the species continuity equation and the energy equations contain derivative terms that must be discretized. A spatially centered finite difference approach applied at each grid point results in one set of linear tridiagonal equations for each of these conservation expressions. Each system can be solved rapidly using the Thomas algorithm for the distribution of one flowfield parameter by holding the other parameters constant during that step in the solution. Continuity, momentum conservation, and the equation of state lead to simple algebraic equations that do not require discretization.

The solution procedure begins with species continuity. The species continuity equation written at each point in the flowfield for a given species produces a system of equations that is solved for the mass fraction of that species at each point. For $n$ species in the flowfield (including ionic species and free electrons), the species continuity equation is solved $n-2$ times, yielding the mass fractions of all the flow species except one of the neutrally charged species and the free electrons. The electron concentration is then obtained through a charge conservation with the ionic species present in the fluid, and the remaining mass fraction is determined by summing the known $n-1$ mass fractions and subtracting from 1 . In this manner, the mass fractions for all species are calculated for the current iteration.

Each energy equation is then solved for its respective temperature distribution. The global energy equation is solved for the translational-rotational temperature distribution, the vibrational energy equation is solved for the vibrational temperature, and the electron-electronic energy equation is solved for the electron-electronic temperature. This procedure gives the three temperature distributions for the current iteration.

Continuity, momentum conservation, and the equation of state are then applied to each grid point. Equation (4) gives the density distribution based upon the current pressure and temperature distributions, Eq. (1) yields the velocity distribution based upon the new density distribution, and Eq. (3) is used to calculate the pressure distribution given the freestream conditions and the local velocity. Solving the flowfield equations in this order, the solution scheme iterates on the solution until the largest change in all flow parameters (density, pressure, temperature, species concentration, and velocity) from one iteration to the next is less than a prescribed tolerance.

\section{Boundary Conditions}

The original axisymmetric code that formed the basis of the normal shock code is a "shock fitting" program. The upstream boundary is just downstream of the shock, and the shock jump conditions provide the upstream boundary conditions for the grid. The shock jump conditions are calculated using the Rankine-Hugoniot relations modified to account for mass diffusion and thermal conduction immediately behind the shock. Including these transport phenomena in the shock boundary conditions is called shock slip and is accomplished by integrating the conservation equations across the shock wave while neglecting collisional terms. The transport phenomena are included due to the high gradients immediately behind the shock front, but collisional terms are neglected because the shock is assumed thin enough that very few collisions occur within the shock wave. Failure to include shock slip results in incorrect boundary conditions and a resulting error in energy conservation in the flow.

Downstream boundary conditions need only be applied to the species continuity equations and the three energy equations. Since the flow is assumed to be in equilibrium at the downstream boundary, the gradients of the species concentrations and temperature distributions are assumed to go to zero. This condition is correct for the nonradiating case and approximate for the radiating case. The radiating case will slowly continue to lose energy through radiation that is not reabsorbed, and so setting the second derivatives of the species concentrations and the temperatures equal to zero at the downstream boundary would be a more accurate approximation for the radiating case. Experience has shown, however, that the calculated flowfield from the shock through the equilibrium region downstream of the shock is not greatly affected by the downstream boundary conditions, even if an unreasonable downstream boundary condition is imposed. The flow solution is affected by this constraint only in the immediate vicinity of the downstream boundary. For these reasons, the simpler zero gradient downstream boundary conditions are used for all cases.

\section{Radiation Modeling}

The radiative heat flux is calculated using a modified version of the program RADICAL, ${ }^{30,31}$ a method which includes atomic continuum radiation, atomic line radiation, molecular band radiation, and free-free Bremsstrahlung radiation for a standard CHON (carbon, hydrogen, oxygen, nitrogen) gas system. The current version of the radiation model includes only those species composed of nitrogen and oxygen. While the original RADICAL code only calculated equilibrium radiation, the version currently employed includes nonequilibrium effects by using the actual species concentrations, the appropriate electron-electronic temperature, and modified forms of the radiation source functions and absorption coefficients. $+23,24,26-28$ This routine calculates the streamwise radiative heat flux term for the global energy conservation equation 
in order to radiatively couple the final solution. Due to the computational intensity of the radiation model relative to the flowfield solver, the radiative flux term is updated once for every 10 flowfield iterations. The current model assumes the radiative term in the electron-electronic energy equation is negligible. Other approximations in the method include the weighting of specific radiation lines to account for the contribution of other lines that are not included explicitly in the solution, the use of average properties for closely spaced lines to save computational time, and the use of exponential approximations for the exponential integral functions in the equation of radiative transfer. The details concerning radiatively coupling the solution are included in Refs. 4 and 27 .

The shock-tube experiments used for comparison in this study measured radiation as viewed from slits in the side of the shock tube, not streamwise radiation as calculated by the original form of the code. Therefore, a model was developed to calculate the radiation intensity incident on the shock-tube wall behind the shock.

Qualitatively, radiant energy transferred through a medium is attenuated through absorption by the medium, but is enhanced by emission from the medium. The absorption depends upon local values for an absorption coefficient, denoted here as $\kappa_{n}$, and the intensity. The emission is given by a source function $S_{\nu}$, defined such that the product $S_{\nu} \kappa_{\nu}$ gives the local emission. The governing expression for radiative transfer in terms of these parameters is given in Eq. (9) ${ }^{32}$ :

$$
\frac{\partial I_{v}}{\partial r}=\kappa_{v}\left(I_{v},-S_{v}\right)
$$

In Eq. (9), $I_{v}$, is the spectral radiation intensity (energy transfer per frequency interval $\mathrm{d} \nu$ at frequency $\nu$ per unit area per unit time per unit solid angle) in the $-r$ direction. This sign convention is illustrated in Fig. 1 as applied specifically to the shock-tube coordinate convention. Choosing $I_{v}$, and $r$ as opposite in direction is convenient when solving for the intensity at a given point. The intensity directed toward the point is found by integrating $\mathrm{Eq}$. (9) over $r$ from the point to an $r_{h}$ where the intensity is known. Under the coordinate system described above, this integration over $r$ goes from $r=0$ to $r=r_{b}$. The solution of Eq. (9) is given by Eq. (10) ${ }^{32}$ :

$$
\begin{aligned}
& I_{\nu}(r=0)=I_{b}\left(r=r_{b}\right) \exp \left(-\int_{0}^{r_{b}} \kappa_{b} \mathrm{~d} r\right) \\
& \quad+\int_{0}^{r_{b}} \kappa_{t}, S_{t} \exp \left(-\int_{0}^{r} \kappa_{t} \mathrm{~d} \hat{r}\right) \mathrm{d} r
\end{aligned}
$$

The desired intensity for modeling the shock-tube output is the intensity incident on the tube wall. Therefore, the ray over which the integration occurs is perpendicular to the flow, with $r=0$ corresponding to the near wall and $r_{b}$ corresponding to the far wall. If the far wall is considered nonemitting and nonreflecting, the $I_{\nu}\left(r=r_{t}\right)$ term in Eq. (10) is zero. Assuming that the shock-tube flow is one dimensional, $S_{v}$ and $\kappa_{\nu}$ are constant over the integration. Therefore, Eq. (10) simplifies greatly, yielding Eq. (11):

$$
I_{t \prime}(r=0)=s_{r}\left(1-e^{\left.-\kappa_{r} r_{b}\right)}\right.
$$

Simply stated, the spectral intensity at frequency $\nu$ incident on the side wall is a simple function of the source function, the absorption coefficient, and the diameter of the shock tube. The spectral intensity $I_{\nu}$, can be integrated over a given frequency range to give the total radiative flux within that range.

$$
I_{\nu_{1}-\nu_{2}}=\int_{\nu_{1}}^{\nu_{2}} I_{\nu} \mathrm{d} \nu
$$
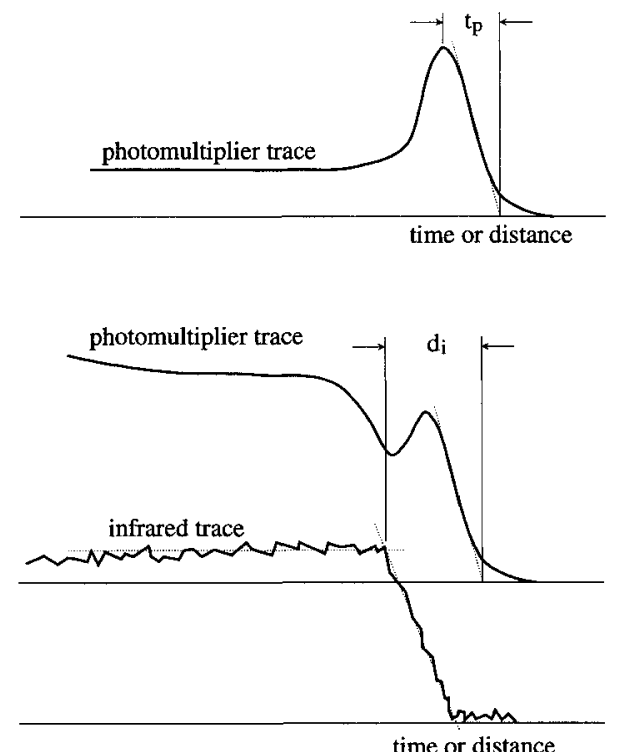

Fig. 2 Time to peak radiation $t_{p}$ and ionization distance $d_{i}$ definitions.

Typical units for $I_{t}$, are $\mathrm{W} /\left(\mathrm{cm}^{2} \mathrm{sr} \mathrm{eV}\right)$; therefore, $I$ can be given in terms of $\mathrm{W} /\left(\mathrm{cm}^{2} \mathrm{sr}\right)$. When considering the intensity of a radiant source of width $r_{b}$, it is often convenient to normalize the intensity by the width $r_{b}$. The results in this report will follow this convention, yielding integrated intensity values with units of $W /\left(\mathrm{cm}^{3} \mathrm{sr}\right)$.

To track the movement of the shock wave down the tube during the experiments, detecting equipment was placed behind windows in the sides of the tube to view radiation intensity incident upon the side wall of the shock tube. The onset of visible radiation was considered to correspond to the arrival of the shock wave. Intensity traces obtained using infrared detectors provided additional information concerning ionization distances and reaction rates. In order to discuss the radiation profiles, characteristic parameters used in describing the radiation front must be defined.

The two primary systems used are shown in Fig. 2. The top illustration corresponds to the data taken from Allen et al. ${ }^{14}$ The Avco study included measurements of time to peak radiation and time to equilibrium radiation. The shock arrival was marked by the onset of visible light (referred to as "luminous front" radiation). The peak nonequilibrium radiation point was the point of maximum intensity within the nonequilibrium region following the shock. A secondary rise in radiation downstream from this zone was attributed to the "attenuation of the shock velocity as it moves down the tube"14; in other words, the shock slows as it proceeds down the tube, resulting in a weaker shock jump later in the test. The fluid shocked earliest was therefore subjected to a shock traveling faster than the speed measured by the test, resulting in higher internal energy in this fluid. A later computational investigation of Wilson's experiment ${ }^{17}$ demonstrated that a similar but more pronounced radiation increase away from the shock seen in Wilson's results is consistent with iron contamination in the shock tube. ${ }^{21}$ The current study does not include iron as a radiator.

The second radiation parameter of interest is the ionization distance $d_{i}$, as defined by Wilson. ${ }^{17}$ The definition of $d_{i}$ is illustrated in Fig. 2. The ionization distance is the distance from the foot of the luminous front (the onset of visible radiation) to the point at which the infrared trace quits rising and becomes relatively level.

\section{Results}

Solutions were obtained for shock speeds between 9.5-12.6 $\mathrm{km} / \mathrm{s}$. Detailed flowfield and radiation data are presented for 
cases compatible with the shock-tube tests of Wilson. ${ }^{17}$ Shocks at $9.5 \mathrm{~km} / \mathrm{s}$ and $0.2 \mathrm{mmHg}, 10.9 \mathrm{~km} / \mathrm{s}$ and $0.1 \mathrm{mmHg}$, and $11.3 \mathrm{~km} / \mathrm{s}$ and $0.1 \mathrm{mmHg}$ were calculated at an initial temperature of $300 \mathrm{~K}$. These experiments were performed in a 6-in.-diam shock tube, and so $r_{b}=6$ in. $=15.24 \mathrm{~cm}$ for the radiation calculation. The path length for the experiment was actually smaller than 6 in. due to the presence of splitter plates in the tube to remove the boundary layer. Since the radiation calculation is normalized by the path length, and since the radiation in the observed frequency range acted optically thin in the test cases, the incorrect path length results in a relatively small error. The computational solutions were obtained using the variable Lewis number diffusion model developed by Gally, ${ }^{3}$ a constant Prandtl number of 0.7 , and a downstream boundary distance $\Delta$ of $6 \mathrm{~cm}$. The computational grid consisted of 101 points with clustering near the ends of the flowfield. Flowfield solution plots in the following section denote every other point in the grid with a symbol to illustrate the grid spacing. Grid and convergence studies for the original stagnation line code showed this grid to be sufficient for a convergence criterion of $(\delta \Phi / \Phi)_{\max }=0.005$ for each parameter $\Phi$ in the flowfield solution. ${ }^{4.5 .27 .28}$ Convergence studies for the current code showed no difference between results for tolerances of 0.005 and 0.001 for the the cases currently under study. All results included in this report were converged to a tolerance of 0.001 .

\section{Flowfield Solutions}

Flowfield solutions for two of the three Wilson cases are presented in Figs. 3 and 4. These figures include temperature distributions and species mole fraction distributions from the shock $(\eta=1)$ to the downstream boundary $(\eta=0)$. The cases are presented in order of increasing shock speed. Thermal nonequilibrium (multiple temperature effects) is evident in the temperature distributions; the translational temperature is quick to respond to the shock, but the vibrational temperature and the electron-electronic temperature climb
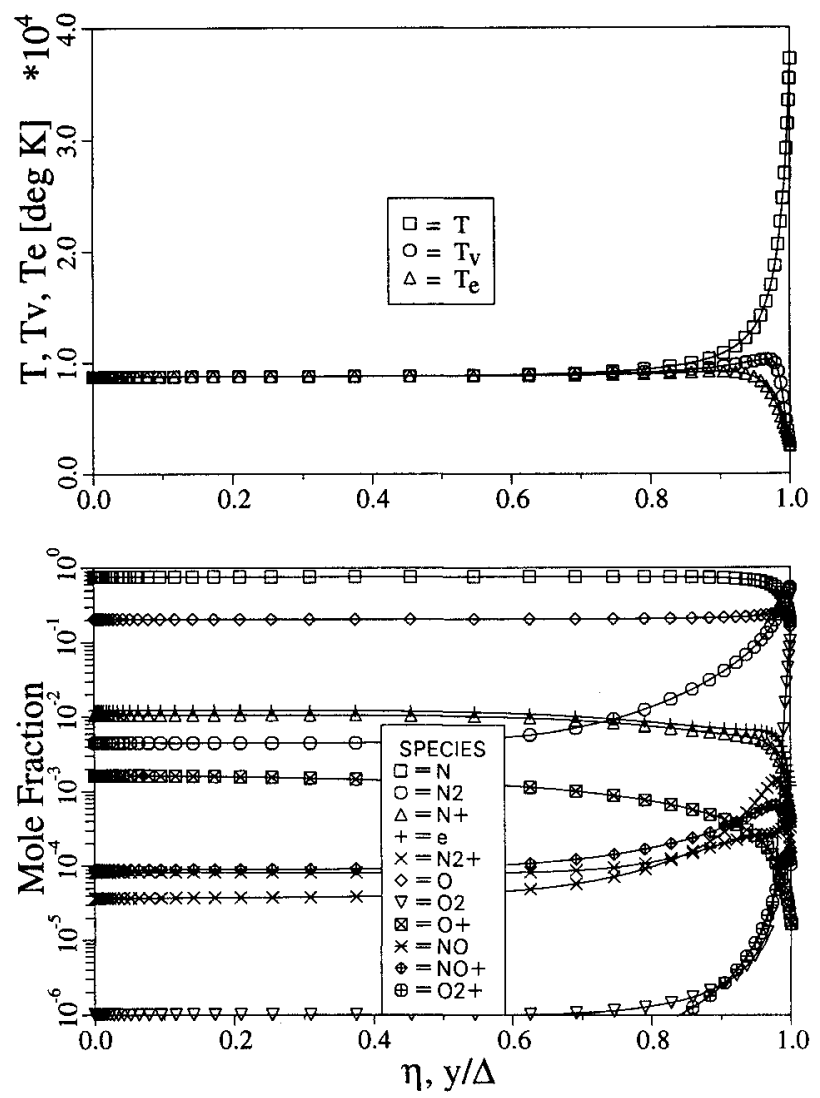

Fig. 3 Temperature and mole fraction curves for the Wilson 9.5$\mathrm{km} / \mathrm{s}, 0.2-\mathrm{mmHg}$ case.
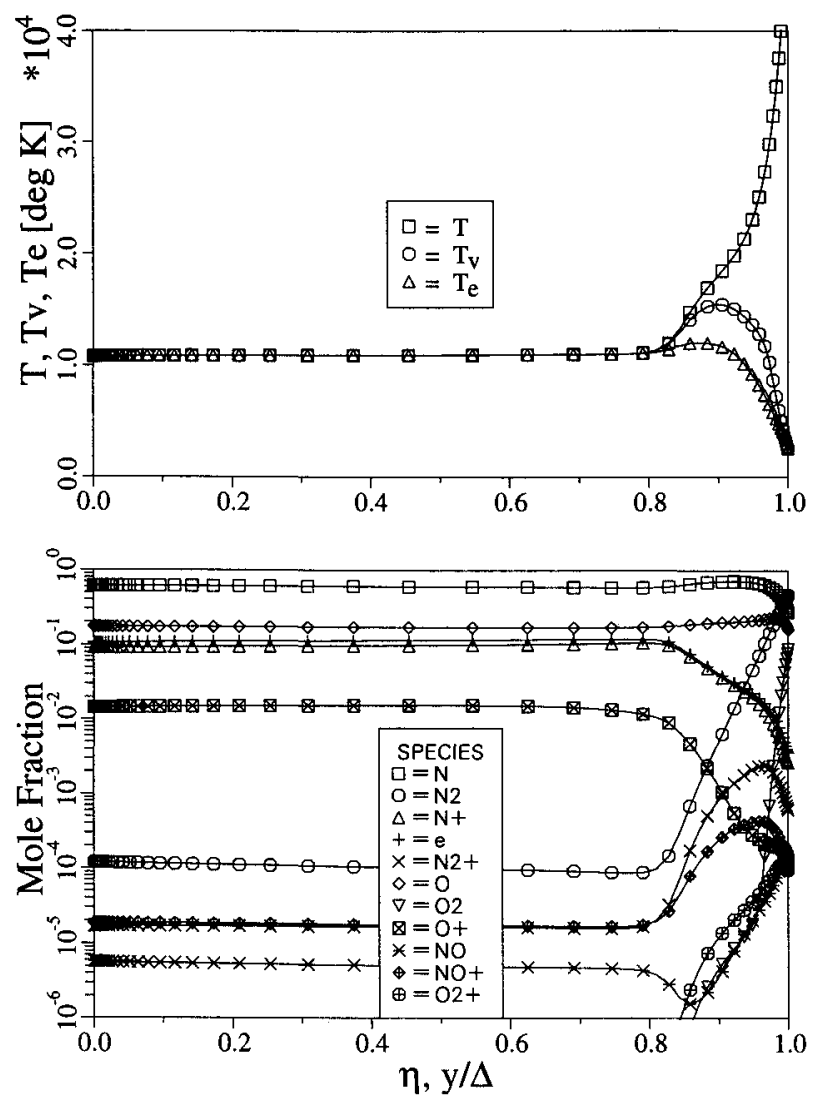

Fig. 4 Temperature and mole fraction curves for the Wilson 11.3$\mathrm{km} / \mathrm{s}, 0.1-\mathrm{mmHg}$ case.

slowly after the shock. This thermal nonequilibrium region is relatively short and decreases in length as the shock speed increases. The nonequilibrium region shortens with increasing shock speed because the stronger shocks produce higher translational temperatures and, hence, higher exchange rates through collisions.

The vibrational and electron-electronic temperatures overshoot the final equilibrium temperature, with this overshoot being more pronounced at the higher shock velocities. The overshoots are due to the dissociation and ionization processes present in the flow. As energy is transferred into the vibrational mode of the diatomic species in the flow, the vibrational temperature increases. After a sufficient amount of energy is transferred into this mode, dissociation will begin to occur. The molecules that are more highly excited vibrationally will be more likely to dissociate than molecules occupying lower vibrational states. In general, a dissociation therefore removes a molecule from the vibrational temperature average that was above the average temperature, and the average vibrational temperature without this particle will therefore be lower. Similarly, electrons that occupy the excited electronic states bring the average electron-electronic temperature for the system up, but these excited electrons are also the ones that are most easily removed in ionization. Thus, the electron-electronic temperature overshoots the proper equilibrium value initially until adequate ionization occurs.

The species mole fraction curves indicate the level of chemical activity present in the flow. The chemical nonequilibrium zone is characterized by gradients in the species concentrations downstream from the shock. The primary species at equilibrium are dissociated nitrogen and oxygen (which together constitute roughly $90 \%$ of the products in all cases), followed on a mole fraction basis by free electrons and then by ionized nitrogen atoms. The equilibrium concentration of $\mathrm{N}_{2}$ drops with increasing shock speed, characterizing the dissociation and ionization processes in the flow. One will note 


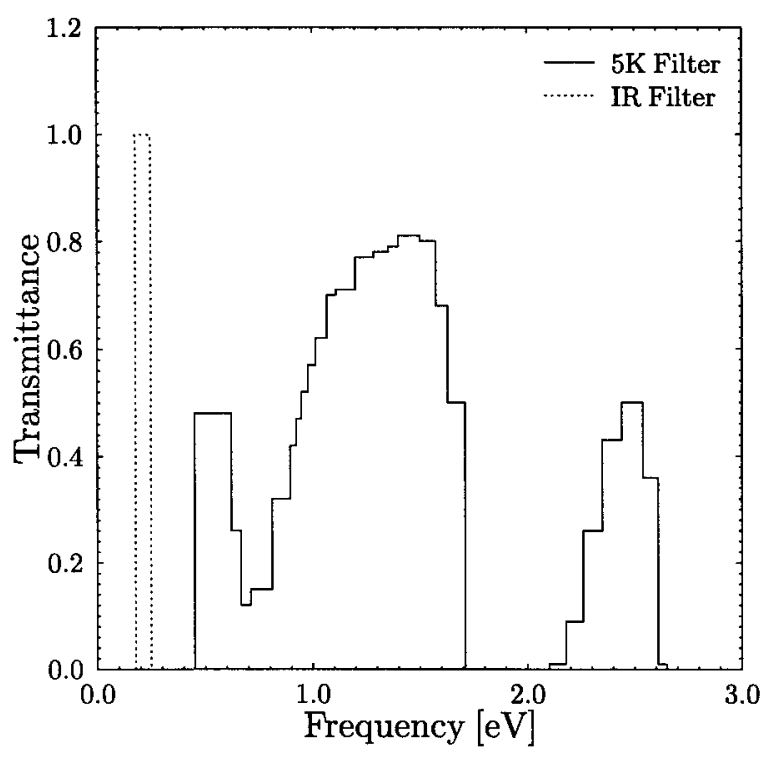

Fig. 5 Filter functions for the Wilson intensity traces.

that the initial concentration of the flow species immediately behind the shock front is not that of standard air due to the mass diffusion effects allowed by the shock slip boundary conditions.

The flowfield solutions illustrate that nonequilibrium exists at Wilson's test conditions. Therefore, Wilson's data provides a means of validating the current nonequilibrium models.

\section{Intensity Traces}

Wilson presents radiation profile data in the form of oscilloscope traces of arbitrary vertical scale. The horizontal axis representing time (or distance down the shock tube) was of a known scale, and so the time to peak radiation and ionization distance can be read directly from the traces. In comparing this experimental data to computational data obtained from the normal shock code, the horizontal axis was scaled appropriately to maintain an accurate time resolution, and the vertical scale of the experimental data was changed to produce the best agreement with the calculated results.

Wilson presents two traces of the incident side wall intensity for the cases he studied. The first trace was observed by a photomultiplier fitted with a combination of a Corning 556 filter and a Corning 371 filter in order to "select a wavelength region around $5000 \AA$ [about $2.5 \mathrm{eV}$ ]." Inspection of the transmittance characteristics of these filters reveals that a significant amount of infrared radiation is also passed through this filter combination..$^{33}$ A composite transmittance function for the filter combination was generated using the spectral response of each of the two filters and is given in Fig. 5. Unfortunately, the spectral response of the photomultiplier used to observe this radiation was not available, but most photomultipliers do respond to some extent to IR radiation. For the present results, significant radiation exists below 2 $\mathrm{eV}$, and this radiation could affect the intensity profiles despite a low photomultiplier response at these frequencies. The traces associated with this filter function will be referred to as the $5 \mathrm{~K}$ traces.

The second trace recorded the infrared radiation between $0.25-0.177 \mathrm{eV}$. Since the specific response of the IR detector was not known, this window was modeled using a filter function with a transmittance of 1 between $0.25-0.177 \mathrm{eV}$ and 0 outside this range. This filter function is also presented in Fig. 5 and the traces taken using this filter function are referred to as the IR traces.

The intensity traces for the Wilson $9.5 \mathrm{~km} / \mathrm{s}$ case are presented in Fig. 6. The $5 \mathrm{~K}$ traces show an initial peak and a secondary rise further downstream. Recall that the experi-
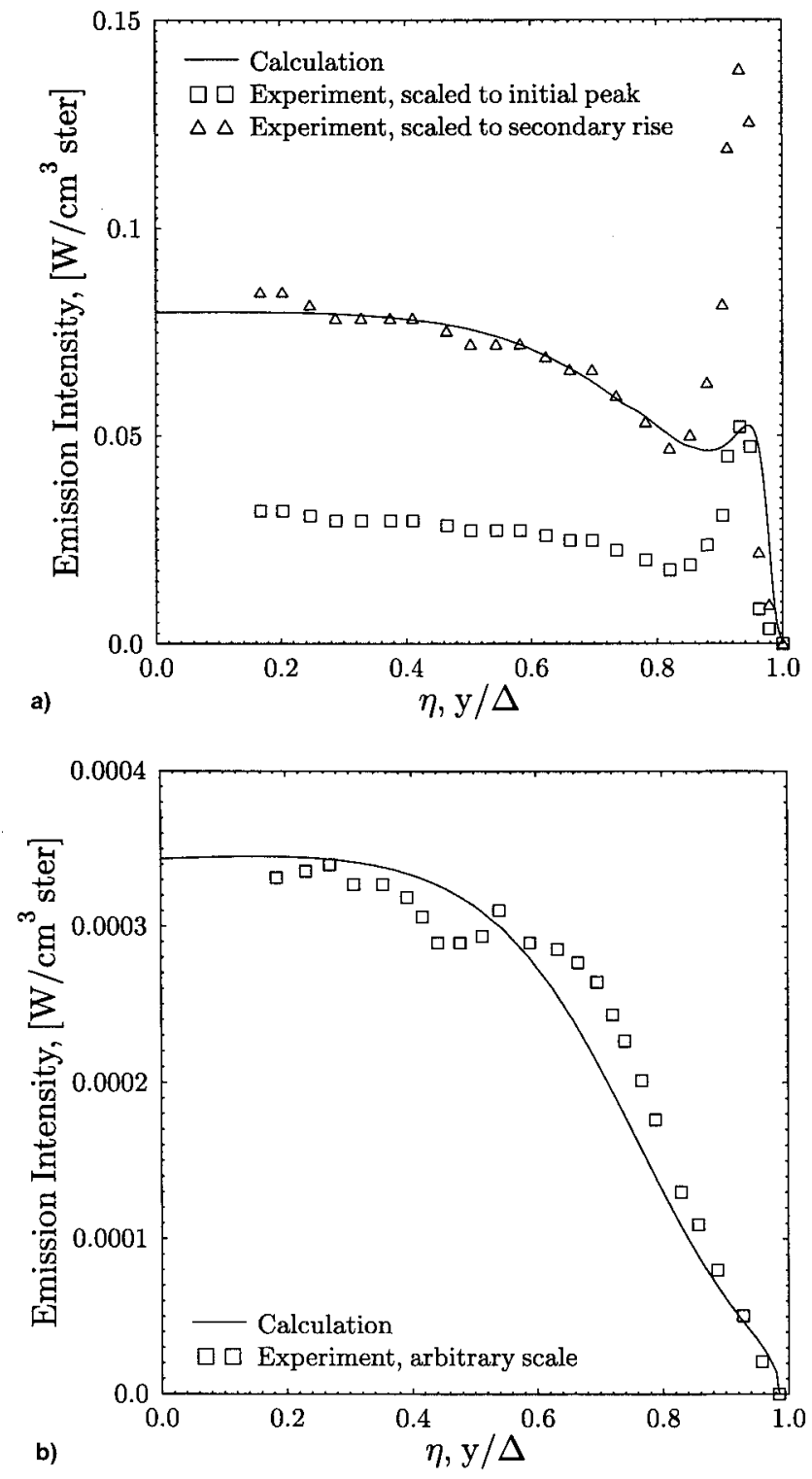

Fig. 6 Intensity traces for the Wilson $9.5-\mathrm{km} / \mathrm{s}, 0.2-\mathrm{mmHg}$ case: a) $5 K$ traces and $b$ ) IR traces.

mental data is scaled arbitrarily; two scales were chosen in Fig. $6 \mathrm{a}$ for the $5 \mathrm{~K}$ data, one to match the magnitude of the initial peak in the calculated distribution and another to match the secondary rise. The initial rise in the computational trace is due primarily to radiation within the filter window centered around $2.5 \mathrm{eV}$. The secondary rise is caused completely by the additional IR radiation allowed to pass by the hump in the filter function near the low eV end. The traces in Fig. 6 illustrate that the two characteristic phenomena are predicted, but scaled incorrectly relative to each other. The IR traces (Fig. 6b) for the $9.5-\mathrm{km} / \mathrm{s}$ case show reasonably good agreement. Both the calculated and the experimental data show a gentle increase in intensity followed by a leveling of the curve to a nearly constant value.

The intensity traces for the Wilson $10.9-\mathrm{km} / \mathrm{s}$ case are presented in Fig. 7. As with the $9.5-\mathrm{km} / \mathrm{s}$ case, the $5 \mathrm{~K}$ traces disagree in the scale of the two primary phenomena. With the proper scalings, the agreement in both areas is good between the calculation and the experiment, especially in the secondary rise and leveling region (Fig. 7a). As with the 9.5$\mathrm{km} / \mathrm{s}$ case, the secondary rise comes completely from radiation passed through the filter function at the low frequency end (namely, between $0.45-1.71 \mathrm{eV}$ ). Both the experimental data and the calculated curve show a gentle decline past the point 

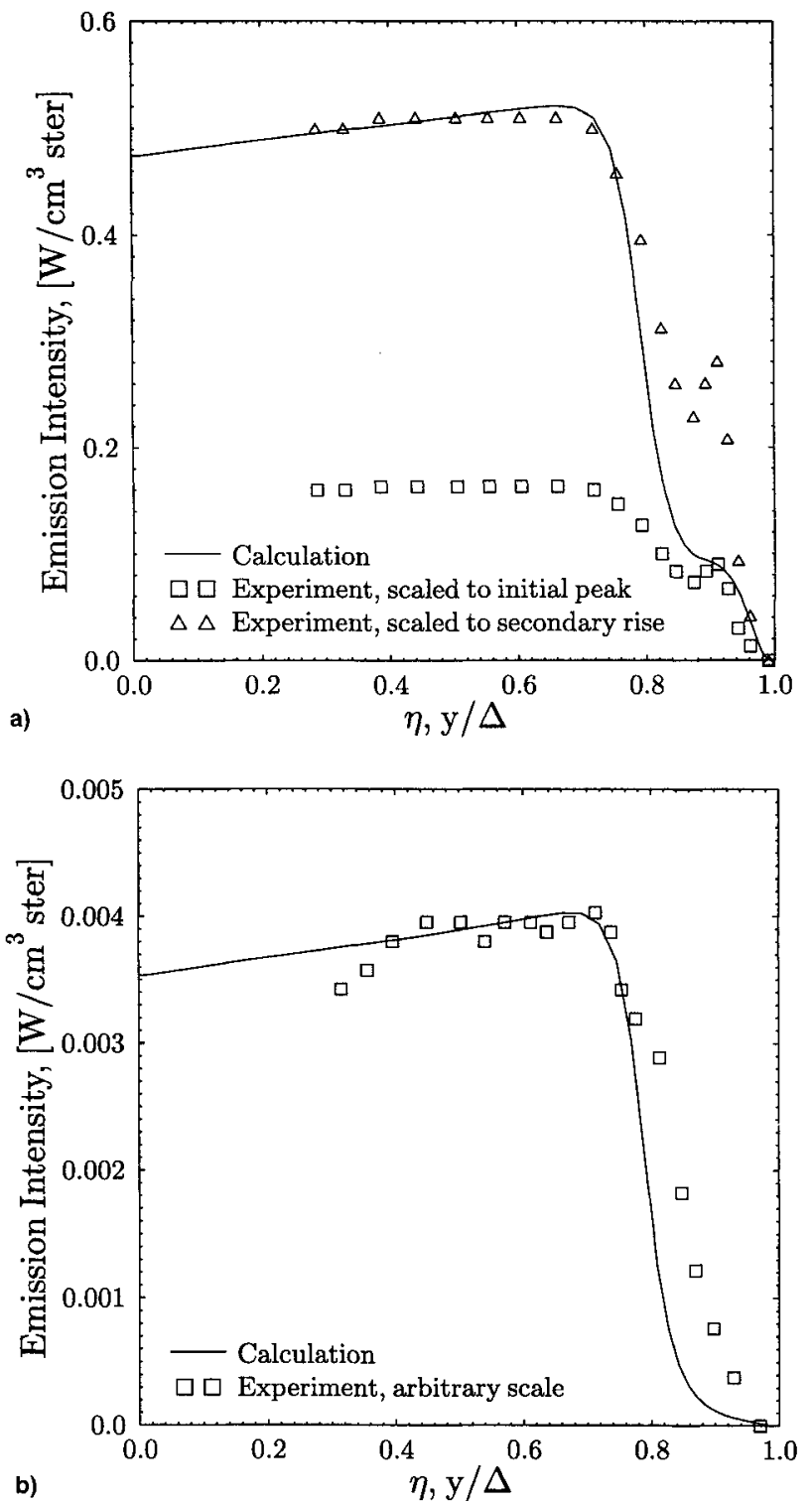

Fig. 7 Intensity traces for the Wilson $10.9-\mathrm{km} / \mathrm{s}, 0.1-\mathrm{mmHg}$ case: a) 5K traces and b) IR traces.

of maximum intensity. Recalling that the horizontal axis is not scaled arbitrarily, the agreement in the time required to reach the plateau for the experimental intensity and the calculated intensity is very good.

The calculated IR trace (Fig. 7b) for the $10.9-\mathrm{km} / \mathrm{s}$ case agrees well with the experimental data. The initial shape of the intensity curves does not match perfectly, but the time from the foot of the intensity curve to the turn at the plateau is the same for the experimental and the calculated data. A gentle drop in both the experimental data and the calculated curve is seen as the curve travels further away from the maximum intensity point.

The final intensity traces included are for the Wilson 11.3$\mathrm{km} / \mathrm{s}$ case, and these traces are presented in Fig. 8. The $5 \mathrm{~K}$ traces again show the same characteristics and the same trend as the traces at 9.5 and $10.9 \mathrm{~km} / \mathrm{s}$. The secondary radiation rise in the $5 \mathrm{~K}$ trace increases in magnitude relative to the initial rise as the shock speed increases, and the initial rise appears as only an inflection point in both the experimental and the calculated curves at $11.3 \mathrm{~km} / \mathrm{s}$. As with the previous $5 \mathrm{~K}$ traces, the initial and secondary rise characteristics are clearly seen in both the calculated trace and the experimental data, but the relative sizes of the phenomena do not match between the experimental data and the calculated results.
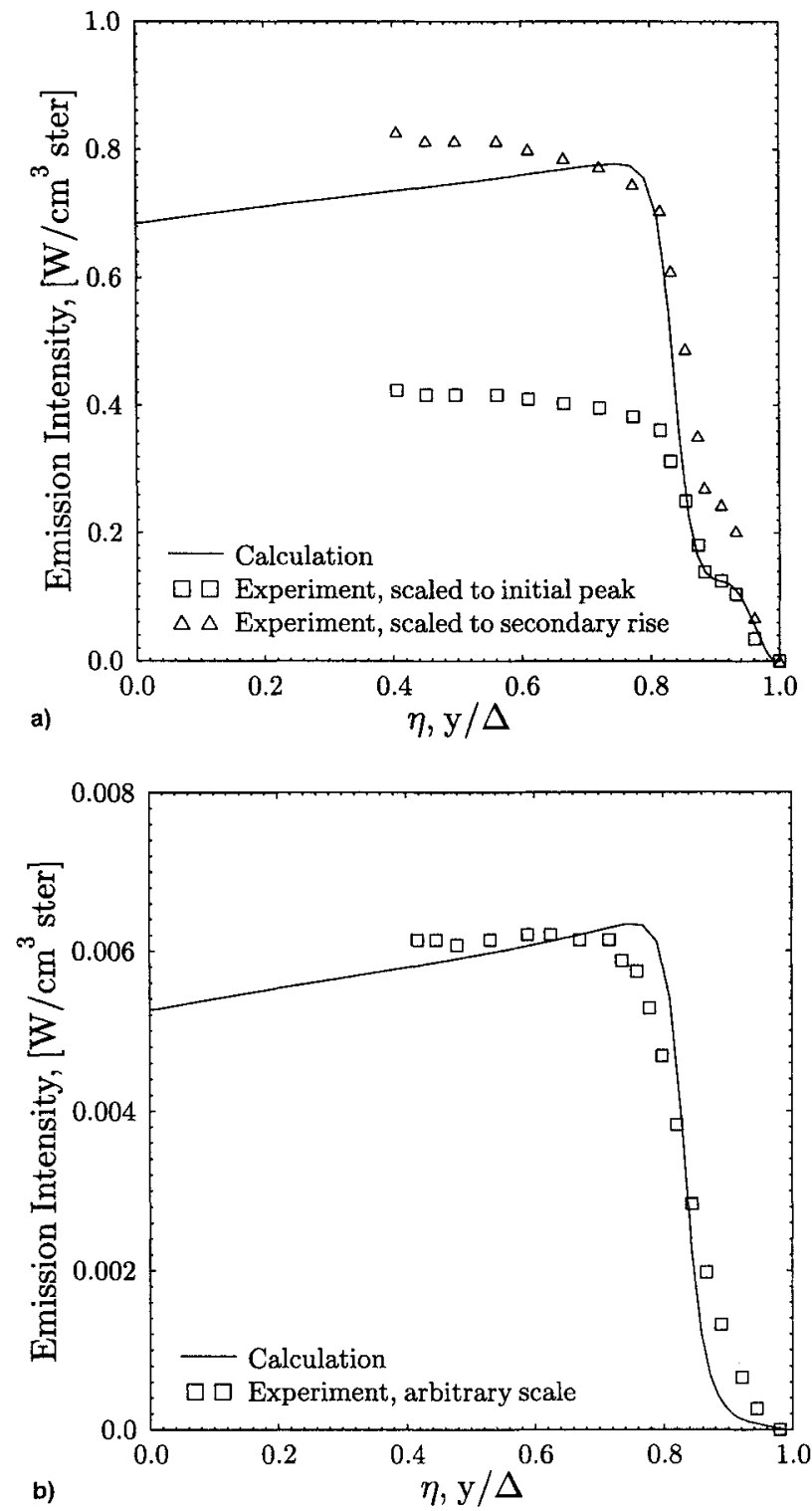

Fig. 8 Intensity traces for the Wilson $11.3-\mathrm{km} / \mathrm{s}, 0.1-\mathrm{mmHg}$ case: a) $5 K$ traces and b) IR traces.

Also, although the time at which the experimental data and the calculated data turn after the secondary rise matches reasonably well, the experimental data continues to rise but the calculated trace drops.

The IR traces for the $11.3-\mathrm{km} / \mathrm{s}$ case are presented in Fig. 8 b. Again, a disagreement is seen in the initial shape of the traces, and a slight difference is seen in the location at which the traces plateau. Despite these differences, good agreement is seen between the experimental results and the calculated trace in general.

Some observations can now be made concerning the characteristics of radiation profiles in nonequilibrium flow. Based upon the calculated and experimental intensity traces presented, it can be concluded that the intensity trace of a single, narrow frequency range is not definitive in determining what regions in the flow have reached an equilibrium radiation level. Secondary peaks in some intensity curves and different behavior between frequency bands prohibit attempts to easily define an equilibrium radiation point. Each experimental Wilson $5 \mathrm{~K}$ trace demonstrates a secondary rise in the radiation after an initial peak. Notice that this secondary increase is also evident in the calculated distributions, even though the simulations include no terms to attenuate the velocity as the shock moves down the tube. The results of Honma and lizuka ${ }^{19}$ 
also support the existence of a secondary rise in the intensity after the initial peak in the nonequilibrium zone. They observed secondary maxima in experimental data for shocks above $10 \mathrm{~km} / \mathrm{s}$ at $0.01 \mathrm{mmHg}$. They then reproduced these secondary peaks computationally using versions of Park's chemistry and radiation models. ${ }^{20.34-36}$ The attenuation of the shock speed as discussed earlier has the effect of generating these secondary intensity increases. However, based upon the experimental and computational data presently available, the secondary rise is a radiative gasdynamic effect and not merely due to limitations in the experimental equipment.

This argument can be extended to the possibility of iron contamination in Wilson's tube. By basing a frequency weighting function on the spectral response curves of the filters used in the experiment, the current calculation includes additional radiation away from $5000 \AA$ and produces this secondary rise without including iron as a radiator. As argued above, the existence of a secondary intensity peak for the radiation is substantiated in both experiments and calculations by various investigators. One should also note that the shock tube section containing the driven gas in Wilson's experiments was aluminum and Pyrex ${ }^{\boxplus 7}$ Additionally, the point at which driver gas and diaphragm contamination arrive is clearly evident in the experimental traces, and the experimental points presented in the current work were taken from the intensity traces prior to the onset of this contamination. Considering these facts, the secondary rise in radiation intensity after what was once thought by some investigators as an equilibrium condition is in fact a gasdynamic occurrence and not merely a result of experimental limitations or contamination.

The remaining unknown in modeling the experimental data of Wilson lies in the photomultiplier spectral response characteristics. The calculated $5 \mathrm{~K}$ traces are consistently misscaled in terms of the phenomena observed between $2.1-2.61 \mathrm{eV}$ and that below $1.71 \mathrm{eV}$. A possible explanation is that the photomultiplier used to observe this radiation had higher response characteristics in the $2.1-2.61 \mathrm{eV}$ range than in the lower range. The IR signal would therefore have been attenuated, reducing the magnitude of the secondary rise in the intensity traces. Unfortunately, the specific photomultiplier used in this experiment is unknown.

This study does not resolve all questions surrounding this set of test cases; approximations and shortcomings in the molecular radiation calculation could also contribute to the improper scaling seen in the intensity results. However, consistency in the trends between the current calculations and Wilson's results, as well as the results of other investigators, strongly support the conclusion that Wilson's dual peak results are indicative of air radiation and not merely a result of contamination.

Investigators use radiation intensity traces to determine time to peak radiation and ionization distance information as defined in Fig. 2. Additional cases at an initial pressure of 0.1 $\mathrm{mmHg}$ and shock speeds of $10 \mathrm{~km} / \mathrm{s}$ and from 11.4 to 12.6 $\mathrm{km} / \mathrm{s}$ were calculated in order to check the correlation between the predicted values for these characteristic times and the experimental results found in Refs. 14 and 17 . Only those cases that allowed for a straightforward application of the definitions shown in Fig. 2 are considered; if the calculated profiles for a given case required interpretation in obtaining $t_{l}$, or $d_{i}$, that case is not included in the following results. The time to peak radiation is presented in Fig. 9. In order to correlate data taken at various initial pressures, the ordinate in Fig. 9 is the product of the initial pressure and the time to peak; the data represented by open triangles are the experimental values taken from Ref. 14, while the filled squares indicate data points generated by the normal shock code. As is seen in the graph, the predictions of the code for time to peak radiation show very good agreement with the data up through $10.2 \mathrm{~km} / \mathrm{s}$, and the results calculated at higher shock speeds appear to perpetuate the trend established by the lower speed data.

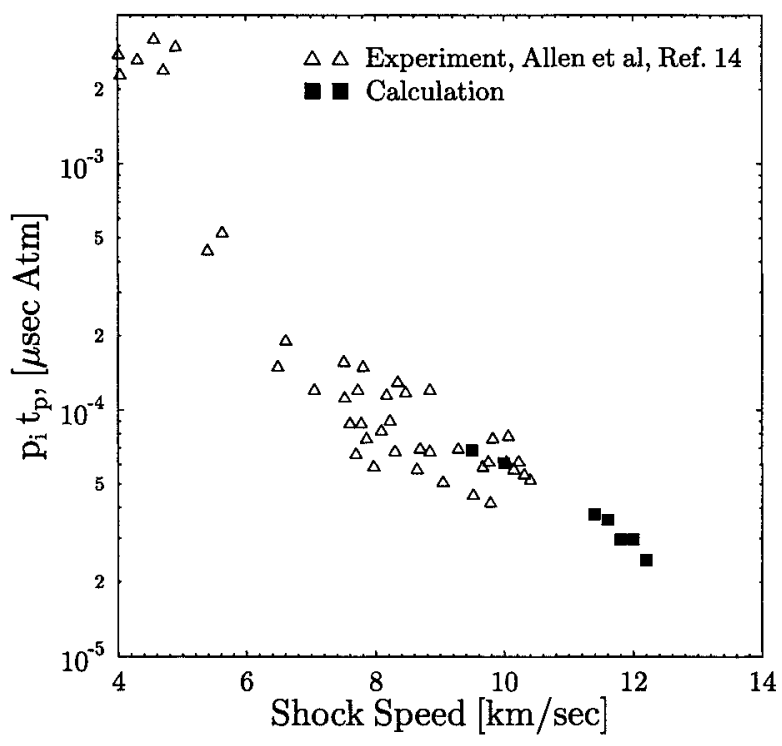

Fig. 9 Time to peak radiation for various shock speeds.

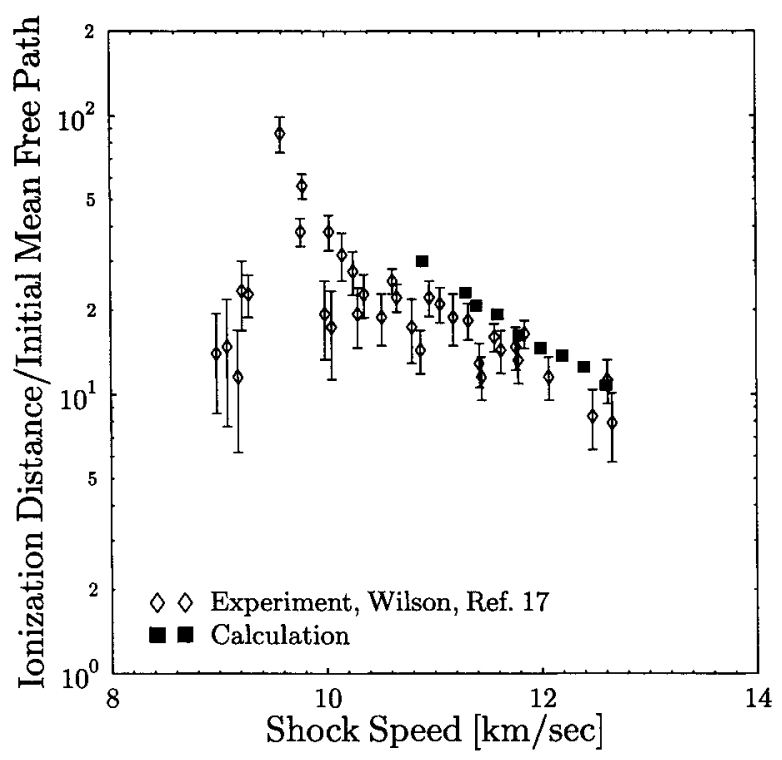

Fig. 10 Ionization distance for various shock speeds.

Ionization distance information is plotted in Fig. 10. The ordinate in Fig. 10 is the ionization distance as defined in Fig. 2 divided by the initial mean free path of the nonshocked air $(0.48 \mathrm{~mm})$. The experimental data included in Fig. 10 is from Wilson, and calculated points between $10.9-12.6 \mathrm{~km} / \mathrm{s}$ obtained by the present study are indicated by filled squares. The calculated ionization distances follow the same trend as the experimental data, staying above the experimental data points, but often falling within the band bounded by the experimental error. In general, this level of agreement is excellent.

\section{Conclusions}

A normal shock version of the viscous shock layer axisymmetric body code was developed to validate current nonequilibrium and radiative models. Calculations were performed for shocks in air at speeds ranging from 9.5 to 12.6 $\mathrm{km} / \mathrm{s}$. The characteristic phenomena seen in the intensity plots of Wilson is reproduced using filter functions derived from the spectral characteristics of the filters used in the experiment. Based upon the current calculations and the experimental and computational work of other investigators, the secondary radiation increase after what many investigators 
had deemed the equilibrium level is a gasdynamic effect and not due to experimental limitations. Ambiguity in the spectral response of the experimental equipment array is a more likely explanation for the difficulties in reproducing Wilson's experimental data than shock speed attenuation or possible iron contamination in Wilson's aluminum and Pyrex ${ }^{\circledR}$ shock tube. Overall, the current model consistently reproduced the experimental data with a level of accuracy that justifies the use of the current models for engineering calculations. Some calculations, such as the ionization distance and the time to peak, show remarkably good agreement with the experimental data.

\section{Acknowledgments}

This work was primarily supported by NASA Grant NAG1-1003 from Langley Research Center, with Lin Hartung Chambers as technical monitor. The first author is supported by an Office of Naval Research Graduate Fellowship administered through the American Society for Engineering Education.

\section{References}

'Miner, E. W., and Lewis, C. H., "Computer User's Guide for a Chemically Reacting Viscous Shock Layer Program," NASA CR2551, May 1975

2Thompson, R. A., "Comparison of Nonequilibrium Viscous Shock Layer Solutions with Windward Surface Heating Data," AIAA Paper 87-1473, June 1987.

'Gally, T. A., "Development of Engineering Models for Nonequilibrium Radiative Phenomena About Aeroassisted Entry Vehicles," Ph.D. Dissertation, Texas A\&M Univ., College Station, TX, May 1992.

${ }^{+}$Gally, T. A., and Carlson, L. A., "An Approximate Local Thermodynamic Nonequilibrium Radiation Model for Air," AIAA Paper 92-2972, July 1992.

"Gally, T. A., Carlson, L. A., and Green, D., "Flowfield Coupled Excitation and Radiation Model for Nonequilibrium Reacting Flows," Journal of Thermophysics and Heat Transfer, Vol. 7, No. 2, 1993, pp. 285-293.

"Gally, T. A., and Carlson, L. A., "User's Guide: Radiation/ Gasdynamic Coupled Hypersonic Nonequilibrium Analysis Code," Texas A\&M Aerospace Engineering Dept., Texas A\&M Univ., TAMRF-6382-94-05, College Station, TX, March 1994.

${ }^{7}$ Moss, J. N., "Reacting Viscous Shock Layer Solutions with Multicomponent Diffusion and Mass Injection," NASA TR-R-411, June 1974.

"Miner, E. W., and Lewis, C. H., "Hypersonic Ionizing Air Viscous Shock Layer Flows over Nonanalytic Blunt Bodies," NASA CR-2550, May 1975.

"Cauchon, D. L., McKee, C. W., and Cornette, E. S., "Spectral Measurements of Gas-Cap Radiation During Project Fire Flight Experiments at Reentry Velocities Near 11.4 Kilometers per Second," NASA TM X-1389, Oct. 1967.

"Greendyke, R. B., and Hartung, L. C., "A Convective and Radiative Heat Transfer Analysis for the Fire II Forebody," AIAA Paper 93-3194, July 1993.

${ }^{11}$ McGough, D. E., Carlson, L. A., and Gally, T. A., "A Preferential Vibration Dissociation Coupling Model for Nonequilibrium Flowfields," AIAA Paper 93-3197, July 1993.

'2Allen, R. A., Keck, J. C., and Camm, J. C., "Nonequilibrium Radiation from Shock Heated Nitrogen and a Determination of the Recombination Rate," Avco Corp. Research Rept. 110, Everett, MA. June 1961 .

1.Teare, J. D., Georgiev, S., and Allen, R. A., "Radiation from the Non-Equilibrium Shock Front," Avco Corp. Research Rept. 112, Everett, MA, Oct. 1961

${ }^{1+}$ Allen, R. A., Rose, P. H., and Camm, J. C., "Nonequilibrium and Equilibrium Radiation at Super-Satellite Re-Entry Velocities,"
Avco Corp. Research Rept. 156, Sept. 1962; also Inst. of the Aerospace Sciences, Paper 63-77, Jan. 1963.

${ }^{15}$ Allen, R. A., "Nonequilibrium Shock Front Rotational, Vibrational, and Electronic Temperature Measurements," Avco Corp. Research Rept. 186, Aug. 1964; also NASA CR-5873.

${ }^{10}$ Wray, K. L., "Excitation Studies on the N2(1+) and the N2 + $(1-)$ Systems in Shock Heated N-N2 Mixtures," Avco Corp. Research Rept. 220, Everett, MA, Aug. 1965.

${ }^{17}$ Wilson, J., "Ionization Rate of Air Behind High-Speed Shock Waves," Physics of Fluids, Vol. 9, No. 10, 1966, pp. 1913-1921.

"Sharma, S. P., Gillespie, W. D., and Meyer, S. A., "Shock Front Radiation Measurements in Air," AIAA Paper 91-0573, Jan. 1991.

1"Honma, H., and Iizuka, H., "Experimental and Numerical Studies of Radiation Emission from High Temperature Air Behind 10 km/s Shock Waves," Society of Automotive Engineers, TP 912025 , 1991.

¿"Park, C., "Assessment of a Two Temperature Kinetic Model for Ionizing Air," AIAA Paper 87-1574, June 1987.

'Park, C., "Review of Chemical-Kinetic Problems of Future NASA Missions, I: Earth Entries," Journal of Thermophysics and Heat Transfer, Vol. 7, No. 3, 1993, pp. 385-398.

:Moreau, S., Bourquin, P. Y., Chapman, D. R., and MacCormack, R. W., "Numerical Simulation of Sharma's Shock Tube Experiment," AIAA Paper 93-0273, Jan. 1993

${ }^{23}$ Carlson, L. A., "Approximations for Hypervelocity Nonequilibrium Radiating, Reacting, and Conduction Stagnation Regions," Journal of Thermophysics and Heat Transfer, Vol. 3, No. 4, 1989, pp. 380-388.

${ }^{2+}$ Carlson, L. A., Bobskill, G. J., and Greendyke, R. B., "Comparison of Vibration Dissociation and Radiative Transfer Models for AOTV/AFE Flowfields," Journal of Thermophysics and Heat Transfer, Vol. 4, No. 1, 1990, pp. 16-26.

${ }^{2}$ Green, D., "A Comparative Study of Vibrational Relaxation Models for the Aeroassisted Orbital Transfer Vehicle Flight Regime," M.S. Thesis, Texas A\&M Univ., College Station, TX, May 1991

${ }^{2 n}$ Carlson, L. A., and Gally, T. A., "The Effect of Electron Temperature and Impact Ionization on Martian Return AOTV Flowfields," Journal of Thermophysics and Heat Transfer, Vol. 6, No. 1, 1991, pp. 9-20.

${ }^{27}$ Carlson, L. A., and Gally, T. A., "Nonequilibrium Chemical and Radiation Coupling, Part I: Theory and Models," Journal of Thermophysics and Heat Transfer, Vol. 6, No. 3, 1992, pp. 385-391.

${ }^{2 *}$ Gally, T. A., and Carlson, L. A., "Nonequilibrium Chemical and Radiation Coupling, Part II: Results for AOTV Flowfields," Journal of Thermophysics and Heat Transfer, Vol. 6, No. 3, 1992, pp. 392-399.

${ }^{29}$ Mott, D. R., "Normal Shock Solutions to the Viscous Shock Layer Equations Including Chemical, Thermal, Thermodynamic, and Radiative Nonequilibrium," M.S. Thesis, Texas A\&M Univ., College Station, TX, Dec. 1993.

"Nicolet, W. E., "User's Manual for the Generalized Radiation Transfer Code (Rad/Equil)," Aerotherm Rept. UM-69-9, Oct. 1969; also NASA CR 116353.

"Nicolet, W. E., "Advanced Methods for Calculating Radiation Transport in Ablation-Product Contaminated Boundary Layers," NASA CR 1656, Sept. 1970.

32Vincenti, W. G., and Kruger, C. H., Introduction to Physical Gas Dynamics, Krieger, Malabar, FL, 1986, p. 463.

${ }^{33}$ Color Filter Glasses, Kopp Glass, Pittsburgh, PA, 1986.

"Park, C., "Calculation of Nonequilibrium Radiation in Flight Regimes of Aeroassisted Orbital Transfer Vehicles," AIAA Paper 84-(0306, Jan. 1984.

s.5ark, C., "A Review of Reaction Rates in High Temperature Air," AIAA Paper 89-1740, June 1989.

36Park, C., "A Fully-Coupled Implicit Method for Thermo-Chemical Nonequilibrium Air at Sub-Orbital Flight Speeds," AIAA Paper 89-1974, June 1989.

${ }^{37}$ Camm, J. C., and Rose, P. H., "Electric Arc-Driven Shock Tube," Physics of Fluids, Vol. 6, No. 5, 1963, pp. 663-678. 